\title{
Delamination of multilayer thermal barrier coatings
}

\author{
Sung Ryul Choi ${ }^{\text {a, John W. Hutchinson }}{ }^{\text {b,* }}$, A.G. Evans ${ }^{b}$ \\ a Department of Mechanical Engineering, Yeungnam University, Kyongsan 712-749, South Korea \\ ${ }^{\mathrm{b}}$ Division of Engineering and Applied Sciences, Harvard University, Pierce Hall, Cambridge, MA 02138, USA
}

Received 11 September 1998; received in revised form 9 March 1999

\begin{abstract}
Multilayer thermal barrier coatings (TBCs) on superalloy substrates are comprised of an intermetallic bond coat, a thermally grown oxide (TGO) layer, and a porous zirconia top coat that provides thermal protection. The TGO attains a thickness of 1-10 $\mu \mathrm{m}$ prior to failure, while the bond coat and zirconia layer are each about 50-100 $\mu \mathrm{m}$ thick. The preferred method for manufacturing TBCs comprises electron beam deposition. This method produces a thin "fully dense" zirconia layer $1 \mu \mathrm{m}$ or $2 \mu \mathrm{m}$ thick between the TGO and the thick "top coat". Edge-delamination and bucklingdelamination are the expected failure mechanisms. Each is addressed. Both occur at the interface between the bond coat and the TGO. Since low in-plane elastic moduli of the porous zirconia layer promote the latter, but suppress the former, there exists a range of moduli wherein both types of failure can be avoided. Two distinct sizes govern buckling-delaminations. Small scale delaminations arise when the TBC top coat has a very low modulus. They have a characteristic size that scales with the thickness of the TGO plus the fully dense zirconia layer: typically tens of microns. In this domain, the dense $\mathrm{TGO} / \mathrm{ZrO}_{2}$ bi-layer buckles by pushing into the thick, more compliant zirconia top layer. The larger scale delaminations occur when the top coat is stiff. They involve not only the bi-layer, but also the zirconia top layer; buckling away from the substrate as a tri-layer. In this case, the total thickness of the TBC determines the extent of the delamination, typically several $100 \mu \mathrm{m}$. (c) 1999 Elsevier Science Ltd. All rights reserved.
\end{abstract}

\section{Introduction}

Thermal barrier coatings (TBCs) consisting of stabilized zirconia are now employed in most turbine engines, permitting gas temperatures to be raised substantially above those for uncoated systems. A detailed description can be found in a recent National Research Council Report (Hillery, 1996). High temperatures are enabled by the low thermal conductivity of the zirconia TBC, coupled

\footnotetext{
* Corresponding author. Tel.: +1 617495 2848; fax: +1 617 495 9837; e-mail: hutchinson@husm.harvard.edu
}

with active cooling of the underlying metal. TBC systems are multilayered. They are designed to inhibit oxidation of the substrate by means of an intermetallic bond coat, as well as provide thermal protection through the TBC itself. The coatings must be able to withstand the mismatch strains generated each time the engine is thermally cycled. TBC durability relies on the integrity of the interfaces: that located between layers as well as that with the substrate. Delamination and spalling are the most common failure modes (DeMasi-Marcin et al., 1989; Lee and Sisson, 1994; Christensen et al., 1996; Sergo and Clarke, 1998; Wang and Evans, 1998; He et al., 1998; Wang and Evans, 1999). 


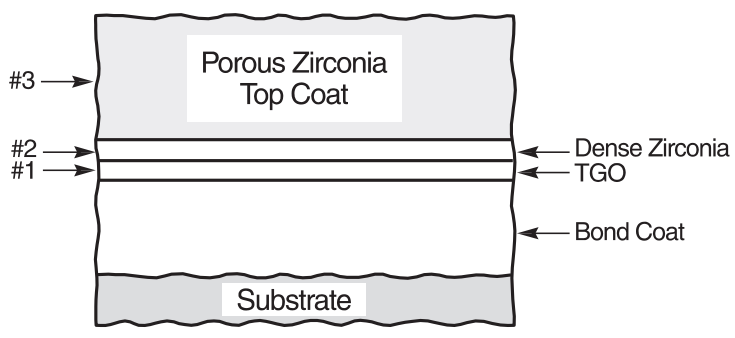

Fig. 1. Layered structure of $\mathrm{TBC}$ considered in this paper.

The layered structure of a representative TBC is shown in Fig. 1. A Ni-based bond coat containing $\mathrm{Al}, \mathrm{Cr}, \mathrm{Co}$ and $\mathrm{Y}$ is applied to the superalloy substrate prior to deposition of the zirconia. Upon exposure at operational temperatures, a thermally grown oxide layer (TGO) forms between the bond layer and the zirconia. The TGO (usually $\mathrm{Al}_{2} \mathrm{O}_{3}$ ) provides the oxidation protection. It is typically in the range $1-10 \mu \mathrm{m}$ when the TBC spalls. Advanced TBCs are made by electron beam deposition (Hillery, 1996). Coatings made by this method have a dense layer of zirconia just above the TGO, with thickness one or two microns. Above this dense layer is a relatively thick porous zirconia layer $(50-100 \mu \mathrm{m})$, the so-called "top coat", which provides the thermal insulation. The columnar grains in this layer have gaps between them that provide in-plane moduli over an order of magnitude smaller than those found for dense $\mathrm{ZrO}_{2}$ (Johnson et al., 1995). ${ }^{1}$ Low moduli are essential to the survival of the TBC as will be explained shortly. Deposition of the zirconia and formation of the TGO both occur at high temperature $\left(\approx 1100^{\circ} \mathrm{C}\right)$. Accordingly, because the coefficients of thermal expansion of the ceramic layers $\left(\alpha_{1}, \alpha_{2}, \alpha_{3}\right)$ are considerably smaller than that of the alloy substrate $\left(\alpha_{s}\right)$, they are subject to in-plane compressive strains in each cooling cycle (stresses on the order of a GPa or more in the TGO and the fully dense zirconia layer (Christensen et al., 1997).

Delamination at the interface between the TGO and the bond coat, with subsequent spalling, is the

\footnotetext{
${ }^{1}$ The low modulus reflects the alignment and morphology of the porosity.
}

chief failure mechanisms for electron beam deposited TBCs (DeMasi-Marcin et al., 1989; Sergo and Clarke, 1998). The failure initiates as an interface separation, which grows by thermomechanical fatigue, accompanied by thickening of the TGO. When the separations become large enough, either large scale buckling or edge delamination are activated. Buckles and delaminations subsequently lead to spalling, wherein the interface crack deflects through the TGO and the TBC up to the free surface (Wang and Evans, 1998; Sergo and Clarke, 1998). The failure is driven primarily by the high compressive stresses in the TGO (Christensen et al., 1996).

Edge-delamination is probable when the inplane moduli of the thick top coat are moderately high, caused by the large elastic strain energy which develops during cooling. For example, a fully dense $100 \mu \mathrm{m}$ zirconia top coat would develop a compressive stress of approximately $1 \mathrm{GPa}$ and an elastic energy per unit area of $250 \mathrm{~J} \mathrm{~m}^{-2}$. This energy density far exceeds the toughness of the interface between the TGO and the bond coat (Wang and Evans, 1998; Sergo and Clarke, 1998), making it virtually certain that the TBC would delaminate (starting either at an edge or from a region of high substrate curvature). On the other hand, high in-plane moduli of the top coat make the TBC less susceptible to buckling at small initial debonds or interface flaws, and therefore can have a beneficial influence in suppressing buckling-delamination. Conditions for buckling-delamination of a multilayer coating will be derived in this paper. Quantitative models for the two competing delamination mechanisms will be employed to estimate the range of in-plane moduli where both mechanisms can be suppressed.

A synopsis of relevant results from the literature for delamination of single layer films will be given in Section 2, serving as background to the present study. Two size domains characterise bucklingdelamination of multilayers. One of these refers to large scale buckles (LSB), wherein the buckle length appreciably exceeds the overall multilayer thickness. The analysis of this domain, presented in Section 3, is applicable to any multilayer. The second domain, addressed in Section 4, operates when the top layer has low in-plane moduli, as is 
the case for many TBCs (Johnson et al., 1995). Now, small scale buckles (SSB) can form, because they can push into the compliant top layer. This buckle size scales with the bi-layer thickness (the TGO plus the dense zirconia layer) rather than with the overall thickness of the TBC.

\section{Delamination of single layer films}

As background for understanding the delamination of multilayer coatings, a brief review of relevant results for single layer films is provided, drawn largely from (Hutchinson and Suo, 1992). This discussion is restricted to considerations of energy release rates relative to interface toughnesses. It neglects the effect of frictional stresses that arise when the crack is strictly mode II (Thouless et al., 1992). This omission is significant because, when friction operates, the fracture behavior is substantially modified, as evident from the analogous problem of debonding and frictional sliding at fiber/matrix interfaces in composites (Hutchinson and Jensen, 1990). Significant friction diminishes the ability of mode II cracks to propagate, sometimes considerably. Accordingly, mode II dominated processes, such as edge-delamination, are less probable than the following analysis would imply. Explicit effects of friction will be addressed in further studies.

For the following discussion, the substrate is assumed to be flat and very thick compared to the film, thickness $t$. Denote the Young's modulus and Poisson's ratio of the film by $E$ and $v$, and the prestress in the film by $\sigma_{0}$, which is assumed to be a uniform equi-biaxial state and taken to positive in compression. Let $\Gamma_{i}(\psi)$ be the mode-dependent toughness of the interface between the film and the substrate (measured in units of $\mathrm{J} \mathrm{m}^{-2}$ ) where the mode mixity is given in terms of the interface crack stress intensity factors by $\psi=\tan ^{-1}\left(K_{\mathrm{II}} / K_{\mathrm{I}}\right)$. The modulus and Poisson's ratio of the substrate are $E_{s}$ and $v_{s}$, respectively.

\subsection{Edge-delamination}

If an interface crack propagates from an edge (Fig. 2(a)), the energy release rate $G$ approaches a

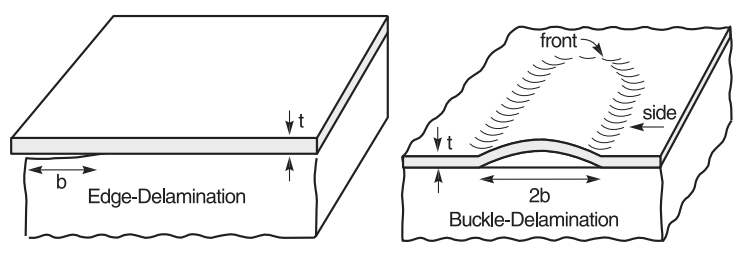

Fig. 2. Delamination modes for compressed films. (a) Edgedelamination; (b) buckling-delaminations: the straight-sided blister.

steady-state, becoming independent of the crack length once it has reached several layer thicknesses. The steady-state interface crack experiences pure mode II at its tip. With the caveat that interface friction is absent so that the crack releases the elastic energy in the layer, the steadystate energy release rate subject to a constraint of plane strain in the direction parallel to the crack front is

$G_{0}=\frac{1-v^{2}}{2 E} \sigma_{0}^{2} t$.

If initial interface edge flaws several film thicknesses in extent are present, then edge-delamination must be expected whenever the combination of pre-stress and film thickness are such that $G_{0}$ exceeds the mode II interface toughness $\Gamma_{i}(\psi)$ $\left(|\psi|=90^{\circ}\right)$. Conversely, if $G_{0}<\Gamma_{i}(\psi)$, insufficient energy is available in the film for an edge-delamination to spread from an edge flaw.

\subsection{Buckle-delamination for straight-sided blisters}

The straight-sided delamination blister shown in Fig. 2(b) propagates as an interface crack at its curved front once it has become fully developed. The precise conditions for the existence of straightsided blisters, as opposed to the more common "telephone cord" morphology wherein the propagating end snakes back and forth, have not yet been discovered (Hutchinson and Suo, 1992; Gioia and Ortiz, 1997). Nevertheless, the straight-sided blister is expected to provide accurate estimates of the size of the initial area of debonded interface required to initiate a buckle delamination as well as the associated energy release rate. Two results will be quoted. The first is the energy release rate at 
the interface crack edge along the straight sides well behind the propagating curved front. The second is the energy release rate along the curved front.

A wide plate of debonded film, width $2 b$ and thickness $t$, clamped along its straight edges undergoes buckling when the compressive stress in the film attains

$\sigma_{\mathrm{c}}=\frac{\pi^{2}}{12} \frac{E}{\left(1-v^{2}\right)}\left(\frac{t}{b}\right)^{2}$.

For compressive film stresses, $\sigma_{0}$, in excess of $\sigma_{\mathrm{c}}$ the film buckles away from the substrate lowering the strain energy stored in the film and producing stress intensities at the interface crack tips. The energy release rate along the straight crack sides of the blister is

$G_{\text {side }}=G_{0}\left(1-\frac{\sigma_{\mathrm{c}}}{\sigma_{0}}\right)\left(1+3 \frac{\sigma_{\mathrm{c}}}{\sigma_{0}}\right)$,

where $G_{0}$ is the same energy density defined in Eq. (2.1). This relation is plotted in Fig. 3(a). Note that $\sigma_{0} / \sigma_{\mathrm{c}}$ increases due either to an increase of or an increase in $b$, which decreases $\sigma_{\mathrm{c}}$. The interface crack tip at the edge of the buckled blister is under mixed mode loading. The strong dependence of $\psi$ on $\sigma_{0} / \sigma_{\mathrm{c}}$ for the right-hand tip is shown in Fig. 3(b). The results plotted are for the case of no elastic mismatch between the film and the substrate. There is some dependence of $\psi$ on the first Dundurs' elastic mismatch parameter,

$\alpha_{\mathrm{D}}=\frac{E /\left(1-v^{2}\right)-E_{s} /\left(1-v_{s}^{2}\right)}{E /\left(1-v^{2}\right)+E_{s} /\left(1-v_{s}^{2}\right)}$,

but little dependence on the second mismatch parameter, $\beta_{\mathrm{D}}$ (Hutchinson and Suo, 1992). As $\sigma_{0} / \sigma_{\mathrm{c}}$ increases, the energy release rate asymptotes to $G_{0}$ after peaking at about 30\% above this limit. The mixity is predominately mode I at the onset of buckling but becomes purely mode II when $\sigma_{0} / \sigma_{\mathrm{c}}$ exceeds about 5. A widening delamination experiences an increase in the proportion of mode II to mode I, arresting when there is a balance between $G_{\text {side }}$ and the interface toughness, $\Gamma_{i}(\psi)$. For metal/ ceramic interfaces, the toughness, $\Gamma_{i}(\psi)$, is significantly larger in near-mode II than in mode I. Consequently, the widening delamination encounters an effectively tougher interface causing it
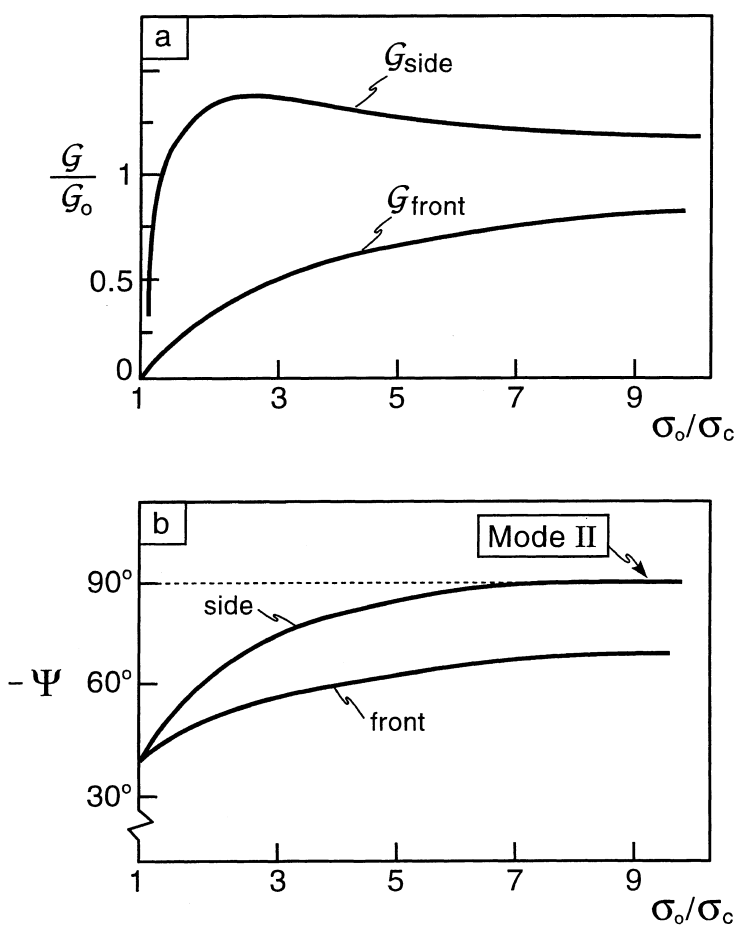

Fig. 3. (a) Energy release rate along the sides and front of the straight-sided blister in a single layer film; (b) mode mixity along the sides and front of a straight-sided blister for the case of no elastic mismatch $\left(\alpha_{D}=0\right)$. The mixity along the front is an approximation based on results from [2] for a circular blister with $v=1 / 3$.

to arrest at a characteristic width. Moreover, there are additional effects of friction in pure mode II.

The energy release rate averaged over the front can be obtained by an integration of Eq. (2.3) with respect to crack width because this gives the energy released per unit of front advance in steady-state (see Hutchinson and Suo (1992) for the derivation):

$$
\begin{aligned}
G_{\text {front }} & =G_{0}\left(1-\frac{\sigma_{\mathrm{c}}}{\sigma_{0}}\right)^{2} \\
& =G_{\text {side }}\left(1-\frac{\sigma_{\mathrm{c}}}{\sigma_{0}}\right)\left(1+3 \frac{\sigma_{\mathrm{c}}}{\sigma_{0}}\right)^{-1} .
\end{aligned}
$$

This result is included in Fig. 3(a), where it can be seen that the energy release rate at the curved front is always less than that along the sides, as also evident from Eq. (2.5). The variation of the mode mixity at the front is shown in Fig. 3(b). 
(This is an approximate estimate obtained using the results in Hutchinson and Suo (1992), wherein $\psi$ is taken to be that for a circular blister of the same radius $b$, with $G=G_{\text {front. }}$ ) It differs significantly from the variation at the sides in that the curve front has a significantly lower proportion of mode II to mode I. Thus, despite the smaller energy release rate, the crack propagates at the front because it experiences a lower effective interface toughness, which more than offsets the energy release rate deficit.

\subsection{Competition between edge-delamination and buckle-delamination for a single layer film}

The energy density quantity $G_{0}$ defined in Eq. (2.1) is central to both delamination mechanisms. The competition between mechanisms is governed by phenomena that occur before steadystate is attained. For edge-delamination, initial interface edge flaws on the order of just several film thicknesses give rise to steady-state with energy release rate equal to $G_{0}$. However, the relevant interface toughness is that for mode II. Conversely, relatively large initial debonded patches are needed at the interface to initiate a buckle-driven delamination. A straight-sided blister begins to buckle up from the substrate when $\sigma_{0} / \sigma_{\mathrm{c}}=1$. By Eq. (2.2), the associated half-width $b$ is given by

$\frac{b}{t}=0.907 \sqrt{\frac{E}{\left(1-v^{2}\right) \sigma_{0}}}$.

For a typical pre-stress level, the half-width is at least ten times the film thickness. If debonded interface patches of this size or greater exist, buckle-driven delamination may initiate. The energy release rate scales with $G_{0}$, as detailed in Fig. 3(a), and the interface toughness will lie somewhere between that for mode I and mode II, depending on $\sigma_{0} / \sigma_{\mathrm{c}}$.

The consequences are as follows. Initiation of buckle-delaminations requires significantly larger interface flaws than is the case for edge-delaminations. On the other hand, the effective interface toughness governing propagation of a buckle-delamination can be significantly lower than that to propagate an edge-delamination due to the mode-dependence of $\Gamma_{i}$. Whether one mechanism predominates over the other depends on details, particularly edge refinements (e.g., tapering or overlapping at the film edge), and the mode dependence of the interface toughness. The fact that buckle-driven delaminations are so commonly observed as the failure mode in highly compressed films reflects both the mode dependence of the interface toughness as well as the friction operating in mode II.

\section{Multilayer films: large delaminations}

In this section the results for delamination of the single layer film are generalized for a film with multiple layers. Similar results have been presented elsewhere within the context of ply delamination of laminates (Nilsson et al., 1993). For long interface cracks, the steady state energy release rate, $G_{0}$, is the same for edge- and buckle-delaminations. The following derivation for buckle-driven delaminations gives $G_{0}$, as well as elucidating other important aspects of buckling in multilayer systems.

As in the case of the single layer, the multilayer is represented as a clamped plate, width $2 b$. This is an accurate representation as long as the buckle width is large compared to the total multilayer thickness, as implied by the terminology, "large". Each layer is assumed to be isotropic with thickness $t_{i}$, Young's modulus $E_{i}$, and Poisson's ratio $v_{i}$. The pre-stress in each layer, $\sigma_{0}^{(i)}$, is uniform and is taken positive in compression.

With the coordinates of Fig. 4, let $y=c$ give the location of the neutral axis of the multilayer for bending about the $z$-axis (see Appendix A). With the pre-buckling stress state as reference, let $\Delta M$ be the change in bending moment about the neutral axis and $\Delta N$ the change in in-plane stress resultant defined by

$$
\begin{aligned}
& \Delta M=-\int_{y_{1}}^{y_{J+1}} \Delta \sigma_{x x}(y-c) \mathrm{d} y \quad \text { and } \\
& \Delta N=\int_{y_{1}}^{y_{J+1}} \Delta \sigma_{x x} \mathrm{~d} y,
\end{aligned}
$$




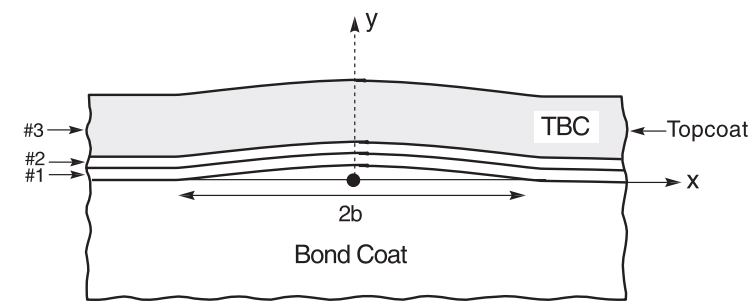

Fig. 4. Geometry of a "large" delamination of a tri-layer coating. The delamination is sufficiently large that the three layers can be regarded as a thin plate in the delaminated region.

where $\Delta \sigma_{x x}$ is the change in stress from the unbuckled, pre-stressed state and $J$ is the number of layers. With $\kappa$ as the curvature and $\Delta \varepsilon$ as the change in stretching strain at the neutral axis (relative to the pre-stressed state), the momentcurvature and stress resultant-strain relations for the changes due to buckling are

$\Delta M=B \kappa \quad$ and $\quad \Delta N=S \Delta \varepsilon$,

where the formulas for the bending stiffness $B$ and the stretching stiffness $S$ are given in Appendix A.

Let the compressive pre-stress resultant be:

$N_{0}=\sum_{i=1}^{J} \sigma_{0}^{(i)} t_{i}$

The critical value of this resultant at which buckling initiates, $\left(N_{0}\right)_{\mathrm{c}} \equiv N_{\mathrm{c}}$, is

$N_{\mathrm{c}}=\left(\frac{\pi}{b}\right)^{2} B$

which reduces to Eq. (2.2) for the case of a single film. The result for the energy release rate generalizing (2.3) is

$G_{\text {side }}=\frac{1}{2 S}\left(N_{0}-N_{\mathrm{c}}\right)\left(N_{0}+3 N_{\mathrm{c}}\right)$.

The average energy release rate at the curved front of the straight-sided blister under steadystate propagation is

$G_{\text {front }}=\frac{N_{0}^{2}}{2 S}\left(1-\frac{N_{\mathrm{c}}}{N_{0}}\right)^{2}$.
A numerical example will be used in Section 5 to illustrate application of these formulas to the tri-layer TBC. Note that variations in compressive pre-stress from layer to layer do not effect the results in Eqs. (3.3)-(3.5), except to the extent that they influence the resultant $N_{0}$.

The limiting energy release rate for $N_{0} \gg N_{\mathrm{c}}$ from both Eqs. (3.4) and (3.5) is

$G_{0}=\frac{N_{0}^{2}}{2 S}$.

As noted above, this is also the energy release rate for edge-delamination of the multilayer, discussed further in Section 6.

\section{Multilayer films: small delaminations}

A TBC with a thick zirconia top coat having low in-plane moduli provides the specific motivation for the analysis in this section. We investigate the possibility of delamination failure at the interface between the bond coat and the TGO with the thin bi-layer plate (TGO and fully dense zirconia) buckling into the compliant zirconia top coat. The limit when the relevant inplane moduli of the top coat become very small is that for the bi-layer plate. The width of the buckle scales with the bi-layer thickness, not with the total TBC thickness. Such delaminations are referred to as "small". The main issue is the level of the in-plane moduli of the top coat necessary to suppress these "small" buckle-delaminations.

The cross-section of the straight-sided buckle delamination is shown in Fig. 5(a). The bond coat is taken as part of the substrate and is not specifically modeled. Layers \#1 and \#2 (the TGO and the thin layer of fully dense zirconia, respectively) are combined as a thin bi-layer plate in the manner of the previous section with $(J=2)$. The case wherein the fully dense zirconia is absent can be obtained as the limit for $t_{2}=0$. The thick top layer (\#3) is analyzed separately, as will be described, and then coupled to the bi-layer plate using the traction-displacement response of layer \#3 in the buckled region (Fig. 5(b)). 

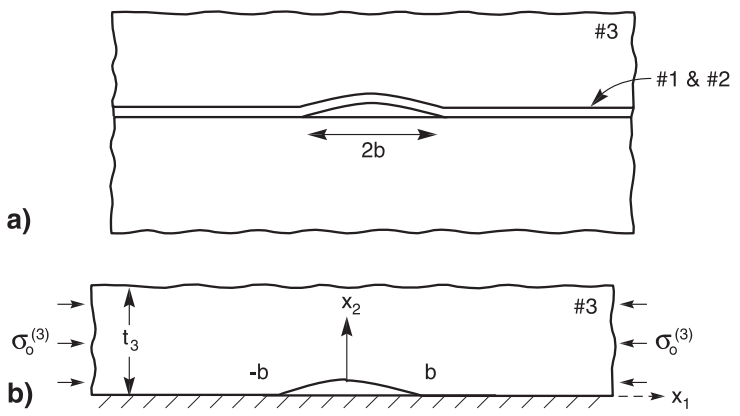

Fig. 5. (a) Geometry for a "small" delamination wherein the bilayer plate buckles into the thick compliant top layer. (b) The problem for determining the restoring stress exerted by the top layer on the bi-layer plate.

Layers \#1 and \#2 are taken to be isotropic and pre-stressed with the same notation used in the previous section. Now, the bending and stretching stiffnesses, $B$ and $S$, are those of the bi-layer plate and are given by expressions in Appendix A (with $J=2$ ). The compressive pre-stress resultant of the bi-layer is

$N_{0}=\sigma_{0}^{(1)} t_{1}+\sigma_{0}^{(2)} t_{2}$.

In the absence of the top layer, \#3, the prestress resultant of the bi-layer at the onset of buckling is (3.3) and the energy release rates are given by Eqs. (3.4) and (3.5).

The thick top coat (\#3) is highly anisotropic due to the porosity between the columnar grains (Johnson et al., 1995). This microstructure also gives rise to low shear moduli governing deflections normal to the plane of the layer. In situ measurements indicate that the (incremental) moduli increase as the temperature imposed on the TBC drops and as the top coat experiences more and more in-plane compression (Johnson et al., 1995). Such behavior would be consistent with pores and micro-cracks closing as the coating is forced to contract. No attempt will be made in this study to model this nonlinear behavior. Here the moduli are taken to be stressindependent and transversely isotropic, with the symmetry axis lying perpendicular to the layer. Specifically, with reference to the coordinate axes in Fig. 5(b), the stress-strain relation in layer \#3 is taken as

$$
\begin{aligned}
& \varepsilon_{11}=\frac{1}{E_{1}^{(3)}} \sigma_{11}-\frac{v_{12}^{(3)}}{E_{1}^{(3)}} \sigma_{22}-\frac{v_{13}^{(3)}}{E_{1}^{(3)}} \sigma_{33}, \\
& \varepsilon_{22}=-\frac{v_{12}^{(3)}}{E_{1}^{(3)}} \sigma_{11}+\frac{1}{E_{2}^{(3)}} \sigma_{22}-\frac{v_{12}^{(3)}}{E_{1}^{(3)}} \sigma_{33}, \\
& \varepsilon_{33}=-\frac{v_{13}^{(3)}}{E_{1}^{(3)}} \sigma_{11}-\frac{v_{12}^{(3)}}{E_{1}^{(3)}} \sigma_{22}+\frac{1}{E_{1}^{(3)}} \sigma_{33}, \\
& \varepsilon_{12}=\frac{1}{2 \mu_{12}^{(3)}} \sigma_{12}, \\
& \varepsilon_{13}=\frac{1}{2 \mu_{13}^{(3)}} \sigma_{13} \quad\left(\mu_{13}^{(3)}=\frac{E_{1}^{(3)}}{2\left(1+v_{13}^{(3)}\right)}\right), \\
& \varepsilon_{23}=\frac{1}{2 \mu_{12}^{(3)}} \sigma_{23} .
\end{aligned}
$$

A compressive pre-buckling stress in layer \#3, $\sigma_{0}^{(3)}$, will also be taken into account.

\subsection{Solution for layer \#3}

For plane strain deformations $\left(\varepsilon_{33}=0\right)$ in the plane of $\left(x_{1}, x_{2}\right)$, Eq. (4.2) can be inverted to give

$\sigma_{11}=c_{11} \varepsilon_{11}+c_{12} \varepsilon_{22}, \quad \sigma_{22}=c_{12} \varepsilon_{11}+c_{22} \varepsilon_{22}$,

$\sigma_{12}=2 c_{44} \varepsilon_{12}$,

where, for the specific cases of interest in this study wherein $E_{2}^{(3)} \gg E_{1}^{(3)}$, the moduli $c_{i j}$ are obtained from Eq. (4.2) in Appendix A. The outcome is

$c_{11}=E_{1}^{(3)} /\left(1-v_{13}^{(3)^{2}}\right), \quad c_{22}=E_{2}^{(3)}, \quad c_{12}=0$,

$c_{44}=\mu_{12}^{(3)} \equiv \lambda c_{11} / 2$,

where $\lambda$ is an independent parameter introduced to scale $\mu_{12}^{(3)}$ with $E_{1}^{(3)}$. For the porous columnar grain structure, $\lambda$ is expected to be between $1 / 2$ and 1 . The equations governing small strain (but nonlinear strain-displacement and equilibrium behavior) for layer \#3 (Fig. 5(b)) are linearized about the uniform pre-buckling stress state. The formulation, which is exact within an incremental plane strain framework, is detailed in Appendix A, along with an outline of the solution method. The layer is infinite in extent in the $x_{1}$ direction, has zero tractions along the top surface at $x_{2}=t_{3}$, and is clamped with zero displacements (relative to the uniform pre-buckling state) along its bottom 
surface outside the buckling region, i.e., on $x_{2}=0$ for $\left|x_{1}\right|>b$.

In principle, it is possible to couple layer \#3 to the bi-layer plate using integral equations to enforce continuity of displacements and tractions between the two regions along $x_{2}=0$ for $\left|x_{1}\right|<b$. We have used an approximate method to achieve the coupling. The approximation makes use of an assumed buckling mode shape in an energy formulation of the buckling problem. The mode shape assumed is that of the bi-layer in the limit wherein it is unconstrained by layer \#3. With $\delta$ as the amplitude of the buckling deflection, the buckling displacement perpendicular to the common interface between layer \#3 and the plate is taken as

$u_{2}=(\delta / 2)\left(1+\cos \left(\pi x_{1} / b\right)\right)$.

The displacement parallel to the common interface, $u_{1}$, is of order $\delta^{2}$, and the energy contribution in layer \#3 arising from this displacement component is very small compared to that from $u_{2}$ and will be neglected. The elastic energy change in layer \#3 from the reference pre-stressed state, $U^{(3)}$, is computed exactly for displacements imposed on $x_{2}=0$ for $\left|x_{1}\right|<b$, where $u_{2}$ is given by Eq. (4.5) and $u_{1}=0$. The steps of the computation are outlined in Appendix A. The result is written as

$U^{(3)}=\frac{1}{2} E_{1}^{(3)} \delta^{2} k\left(\frac{b}{t_{3}}, \frac{\sigma_{0}^{(3)}}{E_{1}^{(3)}}, \frac{E_{2}^{(3)}}{E_{1}^{(3)}}, \lambda, v_{13}^{(3)}\right)$,

where $k$ is a dimensionless function of the parameters listed.

It will be seen that the relevant widths for the small delaminations of the TBC fall well within the range $1 / 3<b / t_{3}<3$. The normalized pre-stress in layer \#3 only influences $k$ when $b / t_{3}$ becomes considerably larger than 3 , as will be illustrated later. For small delaminations, the pre-stress in layer \#3 can be neglected. It has been found that the out-of-plane modulus, $E_{2}^{(3)}$, has virtually no influence on $k$ in the range relevant to the small blisters, even when $E_{2}^{(3)} / E_{1}^{(3)}$ changes by several orders of magnitude (Fig. 6(a)). An asymptotic analysis of the problem for large $E_{2}^{(3)} / E_{1}^{(3)}$ with zero pre-stress is carried out in Appendix A. The result is
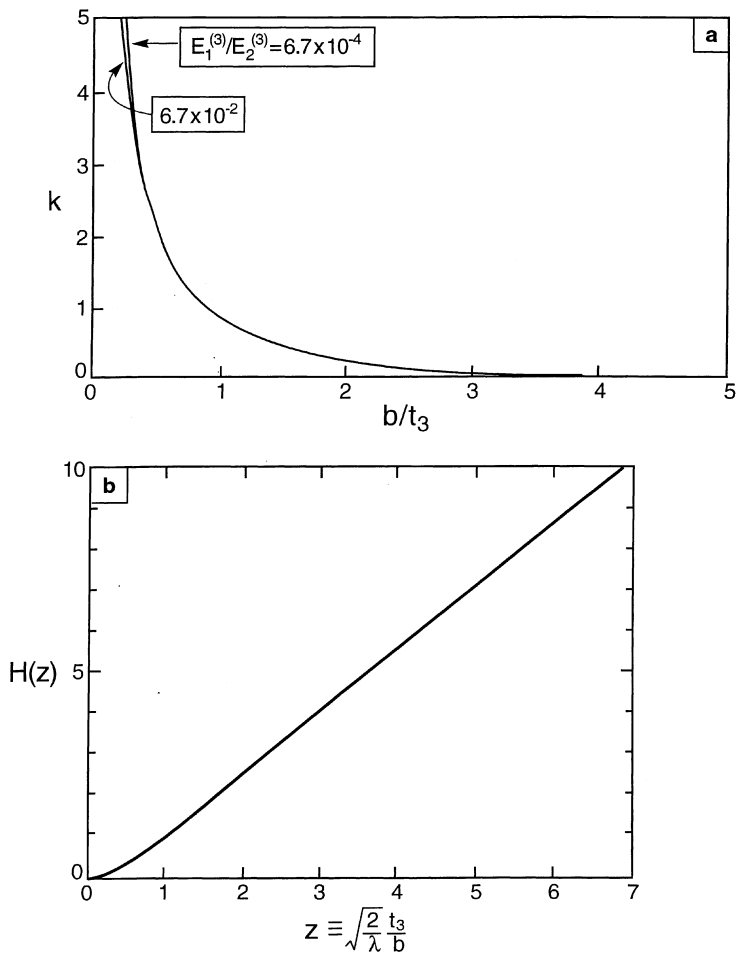

Fig. 6. (a) Curves of $k$ as a function of $b / t_{3}$. (b) The function $H(z)$ for evaluating $k$ from the asymptotic formula (4.7).

$k=\frac{\pi}{2}\left(\frac{\lambda}{2}\right)^{3 / 2} \frac{1}{1-v_{13}^{(3)^{2}}} H\left(\sqrt{\frac{2}{\lambda}} \frac{t_{3}}{b}\right)$,

where the expression for $H$ is given in Appendix A and plotted in Fig. 6(b). For $1 / 3<b / t_{3}<3$, Eq. (4.7) provides $k$ accurately for $E_{1}^{(3)} / E_{2}^{(3)}<0.1$, fully encompassing the range of interest.

\subsection{The solution for the small buckle-delaminations}

The approximate coupled solution (cf. Appendix A) gives the critical value of the compressive pre-buckling stress resultant (4.1) in the bi-layer plate coinciding with the onset of buckling as

$N_{\mathrm{c}}=\left(\frac{\pi}{b}\right)^{2} B+\frac{4 k}{\pi^{2}} E_{1}^{(3)} b$.

For $N_{0}>N_{\mathrm{c}}$ the associated energy release rate of the interface crack lying at the interface between layer \#1 and the substrate (i.e., between the TGO and the bond layer) is 


$$
G_{\text {side }}=\frac{1}{2 S}\left(N_{0}-N_{\mathrm{c}}\right)\left(N_{0}+3 N_{\mathrm{c}}-\frac{16 k}{\pi^{2}} E_{1}^{(3)} b\right) \text {. }
$$

Here, $B$ and $S$ are the bending and stretching stiffnesses for the bi-layer plate $(J=2$ in Appen$\operatorname{dix} \mathrm{A})$. When $k=0$, these formulas reduce to the results obtained for a bi-layer plate in Section 3 in the absence of the thick top layer. The expression for the average steady-state energy release rate on the curved front of the straight-sided delamination cannot be expressed as simply as before. However, for a given value of $N_{0}$ and half-width $b$, it can be evaluated numerically using

$G_{\text {front }}=\frac{1}{b} \int_{b_{0}}^{b} G_{\text {side }} \mathrm{d} b$,

where $b_{0}$ is the half-width associated with the onset of buckling at $N_{0}$, i.e., $b_{0}$ is obtained from Eq. (4.8) with $N_{\mathrm{c}}$ replaced by $N_{0}$.

\section{Buckle-delamination of TBCs: examples}

The number of parameters characterizing a TBC system is too large to present the delamination results comprehensively in graphical form. Instead, results for representative TBCs will be used to illustrate the main features of the phenomenon. The formulas in Sections 3 and 4 are readily programmed to generate results for any set of parameters. Numerical results will be generated for the following TBC systems.

Layer \#1 $\left(\mathrm{Al}_{2} \mathrm{O}_{3}\right)$ :

$t_{1}=1 \mu \mathrm{m}, \quad E^{(1)}=400 \mathrm{GPa}, \quad v^{(1)}=0.2$, $\alpha^{(1)}=8 \times 10^{-6} /{ }^{\circ} \mathrm{C}$,

Layer \#2 (dense zirconia):

$t_{2}=2 \mu \mathrm{m}, \quad E^{(2)}=200 \mathrm{GPa}, \quad v^{(2)}=0.2$, $\alpha^{(2)}=11 \times 10^{-6} /{ }^{\circ} \mathrm{C}$,

Layer \#3 (porous zirconia):

$t_{3}=50 \mu \mathrm{m}, \quad E_{2}^{(3)}=150 \mathrm{GPa}, \quad v_{12}^{(3)}=0$, $v_{13}^{(3)}=0.2, \quad \lambda=1, \quad \alpha^{(3)}=11 \times 10^{-6} /{ }^{\circ} \mathrm{C}$, $E_{1}^{(3)}$ variable.
Additional results are presented for $t_{1}=5 \mu \mathrm{m}$ and for $t_{3}=100 \mu \mathrm{m}$ in order to illustrate important trends. The effect of the in-plane modulus of layer $\# 3, E_{1}^{(3)}$, will be studied by varying the ratio, $E_{1}^{(3)} / E_{2}^{(3)}$. The out-of-plane modulus, $E_{2}^{(3)}$, has been reduced slightly below the fully dense value for zirconia to reflect the effect of the porosity. Note that the shear modulus in layer \#3 relevant to the plane strain deformation is $\mu_{12}^{(3)} \cong E_{1}^{(3)} / 2$. The substrate is considered to be a representative $\mathrm{Ni}$ superalloy with $\alpha_{s}=14 \times$ $10^{-6} /{ }^{\circ} \mathrm{C}$ and very thick relative to the TBC.

\subsection{Small and large buckle-delaminations}

For the TBC system having properties specified in Eq. (5.1), we first consider the temperature drop necessary to bring an initial straight-sided flaw of width $2 b$ to the onset of buckling. Specifically, for the purpose of illustration, assume that the stresses in all three layers are zero at $T=1000^{\circ} \mathrm{C}$, and assume the coefficients of thermal expansion for the layers and for the substrate are temperatureindependent with the values listed above. For a temperature drop, $\Delta T$, measured from $1000^{\circ} \mathrm{C}$, the compressive pre-buckling stress in each of the layers is given by

$\sigma_{0}^{(i)}=\frac{E^{(i)} \Delta \alpha^{(i)} \Delta T}{\left(1-v^{(i)}\right)}$,

where $\Delta \alpha^{(i)}=\alpha_{s}-\alpha^{(i)}$, and for layer \#3 $E^{(i)}$ and $v^{(i)}$ are the in-plane quantities, $E_{1}^{(3)}$ and $v_{13}^{(3)}$. Fig. 7 presents the temperature drop required to initiate buckling as a function of the half-width of the interface flaw, $b$. Two sets of predictions are shown, one for $E_{1}^{(3)}=0.01 \mathrm{GPa}$ and the other for $E_{1}^{(3)}=0.05 \mathrm{GPa}$. The solid-line curves are obtained using Eq. (4.8) with full accounting for the dependence of $k$ on $\sigma_{0}^{(3)} / E_{1}^{(3)}$. The dashed-line curves are obtained using the tri-layer plate result (3.3): Note that its accuracy deteriorates for smaller flaws and large in-plane moduli. The smallest flaws that buckle for $\Delta T=1000^{\circ} \mathrm{C}$ have a halfwidth of about $40 \mu \mathrm{m}$, but only if the top coat has a very low in-plane modulus. Larger flaws require less temperature drop to initiate buckling. In passing, we mention that a TBC with a fully dense $50 \mu \mathrm{m}$ top coat would only buckle for 


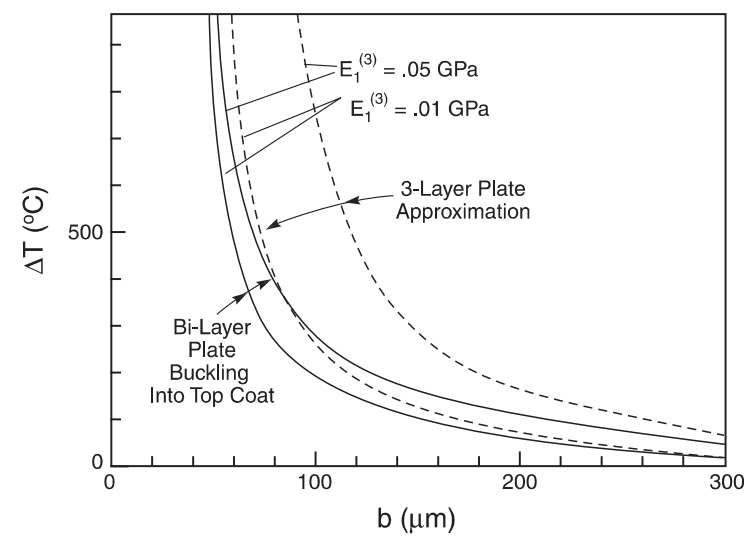

Fig. 7. The temperature drop required to initiate buckling of a straight-sided blister of half-width $b$. The properties of the trilayer TBC are specified in Eq. (5.1). Results are shown to for two values of the in-plane modulus of the top coat. The solid line curves are based on the accurate analysis using Eq. (4.8); the dashed-line curves are based on the tri-layer plate approximation (3.3) for large delaminations.

$\Delta T=1000^{\circ} \mathrm{C}$ if the half-width of the flaw exceeded about $800 \mu \mathrm{m}$.

\subsection{Energy release rates: the role of the in-plane} modulus of layer \#3

For a temperature drop $\Delta T=1000^{\circ} \mathrm{C}$, the compressive pre-stresses in layers \#1 and \#2 (based on Eq. (5.1)) are

$\sigma_{0}^{(1)}=3.0 \mathrm{GPa}$ and $\sigma_{0}^{(2)}=0.75 \mathrm{GPa}$.

For the small values of $E_{1}^{(3)} / E_{2}^{(3)}$ considered in the example which follows, the pre-stress in layer \#3 has no influence either on the elastic energy stored in the TBC or on buckling. The value of the pre-stress resultant (4.1) for a $1 \mu \mathrm{m}$ thick TGO is $N_{0}=4.5 \mathrm{kNm}^{-1}$ and the limiting value of Eqs. (3.4) and (3.5) for $N_{0} \gg N_{\mathrm{c}}$ is

$G_{0}=N_{0}^{2} /(2 S)=12.15 \mathrm{~J} \mathrm{~m}^{-2}$.

This steady-state level is increased to $56 \mathrm{~J} \mathrm{~m}^{-2}$ when the TGO thickens to $5 \mu \mathrm{m}$.

Curves of the energy release rate computed from Eqs. (4.9) and (4.10) are plotted in Fig. 8 as a function of the half-width $b$ for various values of the moduli ratio $E_{1}^{(3)} / E_{2}^{(3)}$. For values of this ratio

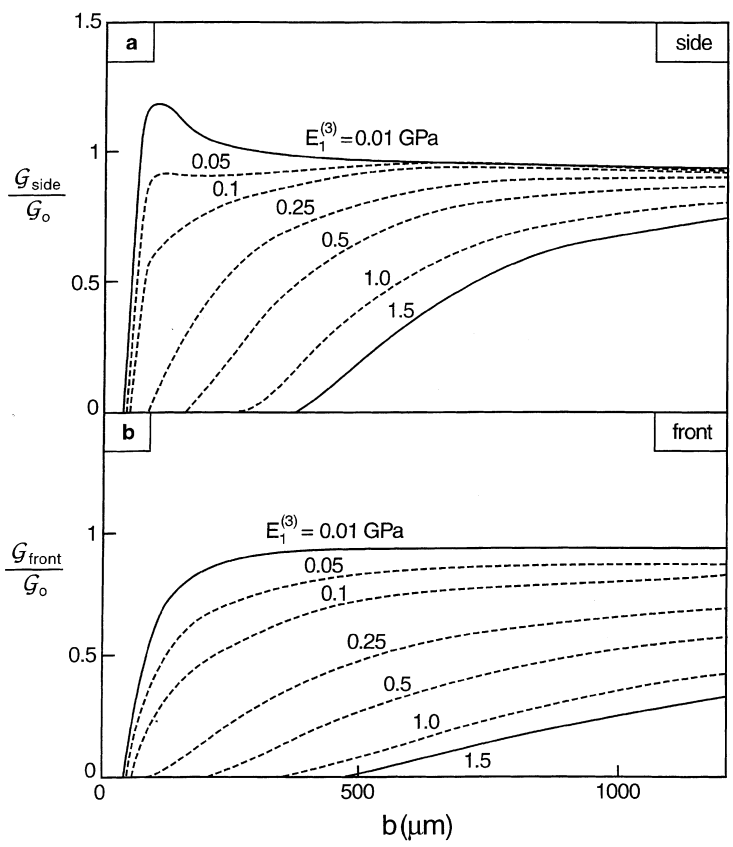

Fig. 8. Energy release rates for the straight-sided blister in a TBC specified by Eq. (5.1) for various values of in-plane modulus of the top coat at a temperature drop of $1000^{\circ} \mathrm{C}$. (a) Along the sides; (b) along the front.

less than about $10^{-4}$, the in-plane modulus of layer \#3 has no influence and the energy release rate is the same as it would be in the absence of the thick top layer. Consequently, buckles with half-widths greater than about $50 \mu \mathrm{m}$ have energy release rates on the order of $G_{0}$. However, when $E_{1}^{(3)} / E_{2}^{(3)}$ exceeds about $10^{-3}$, the top layer constrains the amplitude of the buckle and significantly lowers its energy release rate. For $E_{1}^{(3)} / E_{2}^{(3)}=3 \times 10^{-3}$, buckles with a half-width less than $300 \mu \mathrm{m}$ are completely suppressed. Accordingly, a $50 \mu \mathrm{m}$ top layer having in-plane moduli $E_{1}^{(3)} / E_{2}^{(3)}>3 \times 10^{-3}$ is sufficient to avert small buckle-delaminations. This is the range found in practice for TBCs (Johnson et al., 1995).

The mode mixity, $\psi$, associated with the energy release rate along the side of the blister is shown in Fig. 9. As the width of the buckle increases, crack tip conditions rapidly approach mode II. Mode mixity along the curved front has not been determined, but it will have a more substantial mode I component. As already noted, spreading of the 


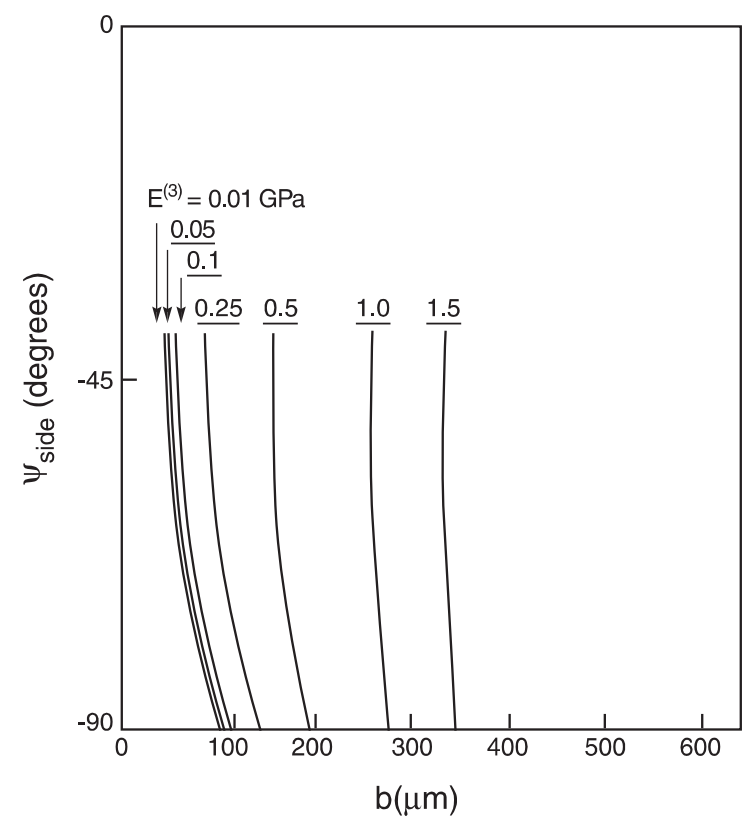

Fig. 9. Mode mixity associated with the interface crack along the sides of the blister for the example in Fig. 8.

buckle-delaminations will be affected by the modedependence of the interface toughness and the friction.

Energy release rates are plotted in Fig. 10 for an example identical in all respects to the former ex-

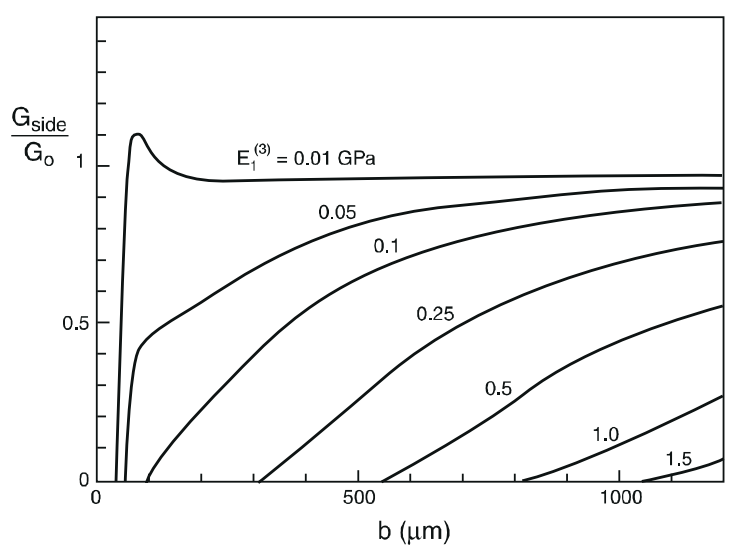

Fig. 10. Energy release rates for the straight-sided blister in a TBC which is identical to that in Fig. 8 except that the top layer thickness is $t_{3}=100 \mu \mathrm{m}$ rather than $t_{3}=50 \mu \mathrm{m}$. cept that now the thickness of the top coat is $t_{3}=100 \mu \mathrm{m}$. At a given moduli ratio, $E_{1}^{(3)} / E_{2}^{(3)}$, the thicker top coat is more effective at suppressing buckling and lowering the energy release rate.

\section{Edge-delamination versus buckle-delamination}

Except when $E_{1}^{(3)} / E_{2}^{(3)}$ is small, the top coat contributes to the energy available for delamination, $G_{0}$. At the same time, however, it makes it less likely that small delaminations will buckle. It has already been noted that $G_{0}$ defined in Eq. (3.6) represents the limiting energy release rate for the buckle-delaminations as they grow. For the TBC, $G_{0}$ is also the steady-state energy release rate for edge-delamination along the interface between the TGO and the bond coat, absent frictional interaction along the interface crack faces. The crack is closed and the delaminated film remains flat (until its length reaches the point where the unrelaxed component of stress parallel to the edge produces edge-buckles (Thouless et al., 1994)). The edgedelamination crack is subject to pure mode II conditions.

The energy density $G_{0}$ is a central quantity in assessing the likelihood of both edge- and buckledelaminations. If the interface toughness, $\Gamma_{i}(\psi)$, exceeds $G_{0}$, delaminations will not occur. Recall that the relevant toughness for buckle-delaminations will be much less than that for edge-delaminations due to mode mixity and frictional effects. A plot of $G_{0}$ for edge-delaminations from Eq. (3.6) as a function of $E_{1}^{(3)} / E_{2}^{(3)}$ is given in Fig. 11(a) for the examples discussed in Section 5 for $\Delta T=$ $1000^{\circ} \mathrm{C}$. At $E_{1}^{(3)} / E_{2}^{(3)}$ less than about $10^{-3}$, the energy release rate $\left(G_{0} \cong 12.5 \mathrm{~J} \mathrm{~m}^{-2}\right)$, is comprised almost entirely of the elastic energy in layers \#1 \& $\# 2$, with most of energy in layer \#1. Sharp increases in $G_{0}$ occur once $E_{1}^{(3)} / E_{2}^{(3)}$ exceeds a critical level (depending on $t_{3}$ ), corresponding to storage of significant elastic energy in the top coat. In practice, TBCs have moduli in the range $\left(E_{1}^{(3)} / E_{2}^{(3)}>10^{-2}\right)$ wherein the top coat contributes significantly to the energy release rate.

The beneficial effect of larger $E_{1}^{(3)} / E_{2}^{(3)}$ in suppressing the buckling of small initial flaws is illustrated in Fig. 11(b). Here, the smallest flaw 


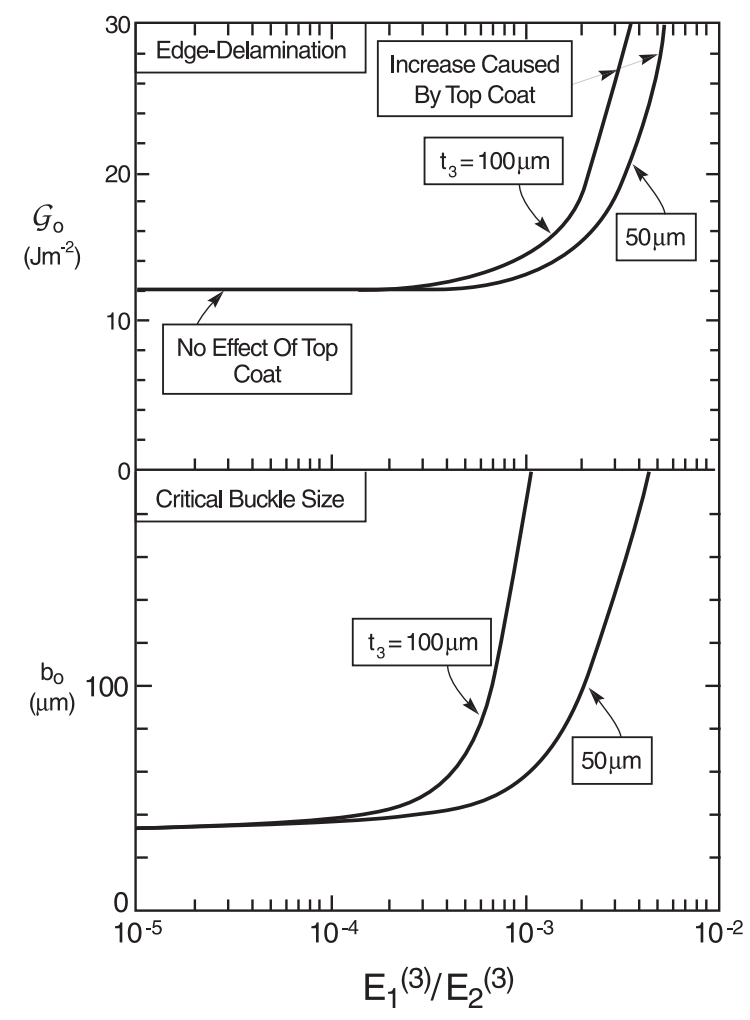

Fig. 11. (a) Energy release rate quantity $G_{0}$ as a function of $E_{1}^{(3)} / E_{2}^{(3)}$. (b) Half-width $b_{0}$ of the smallest straight-sided blister which will buckle as a function of $E_{1}^{(3)} / E_{2}^{(3)}$. Both figures apply to the TBC specified in Eq. (5.1).

half-width, $b_{0}$, which will buckle is plotted against $E_{1}^{(3)} / E_{2}^{(3)}, \quad\left(\Delta T=1000^{\circ} \mathrm{C}\right)$. The half-width, $b_{0}$, corresponds to the size of the blister at the onset of buckling; it is given by Eq. (4.8) with $N_{0}$ replacing $N_{\mathrm{c}}$. At values of $E_{1}^{(3)} / E_{2}^{(3)}$ less than $10^{-4}$ the top coat exerts effectively no constraint on buckling of the bi-layer. At higher values of $E_{1}^{(3)} / E_{2}^{(3)}$, the top coat constrains buckling and those delaminations susceptible to buckling are larger. Note that these buckles still lie within the regime wherein the bi-layer plate buckles into the compliant top coat; they are not the large buckles of Section 3 until the half width is larger than about $300 \mu \mathrm{m}$.

The countervailing trends in Fig. 11 can be brought together to highlight fail-safe conditions wherein initial delaminations will neither spread nor buckle. First, consider the condition such that edge-delaminations will not spread. Combinations of $t_{3}$ and $E_{1}^{(3)} / E_{2}^{(3)}$ corresponding to $G_{0}=\Gamma_{\mathrm{II} i}$, where $\Gamma_{\mathrm{II} i}$ is the mode II interface toughness, are plotted as dotted lines on Fig. 12(a) and 12(b). A top coat with a combination of thickness and inplane modulus lying to the left of each curve (for the relevant $\Gamma_{\mathrm{II} i}$ ) will not experience steady-state edge-delamination. Otherwise it will spontaneously delaminate (unless it is arrested by friction). Next, combinations of $t_{3}$ and $E_{1}^{(3)} / E_{2}^{(3)}$ that avert buckling of pre-existing delaminations of halfwidth $b_{0}$ have been plotted as solid lines on Fig. 12(a) and 12(b). Such combinations lie to the right of each curve for the given initial flaw size, which corresponds to the onset of buckling. Combinations of $t_{3}$ and $E_{1}^{(3)} / E_{2}^{(3)}$ which exclude both edge-delamination and buckling clearly exist, depending on the initial flaw size and the in-

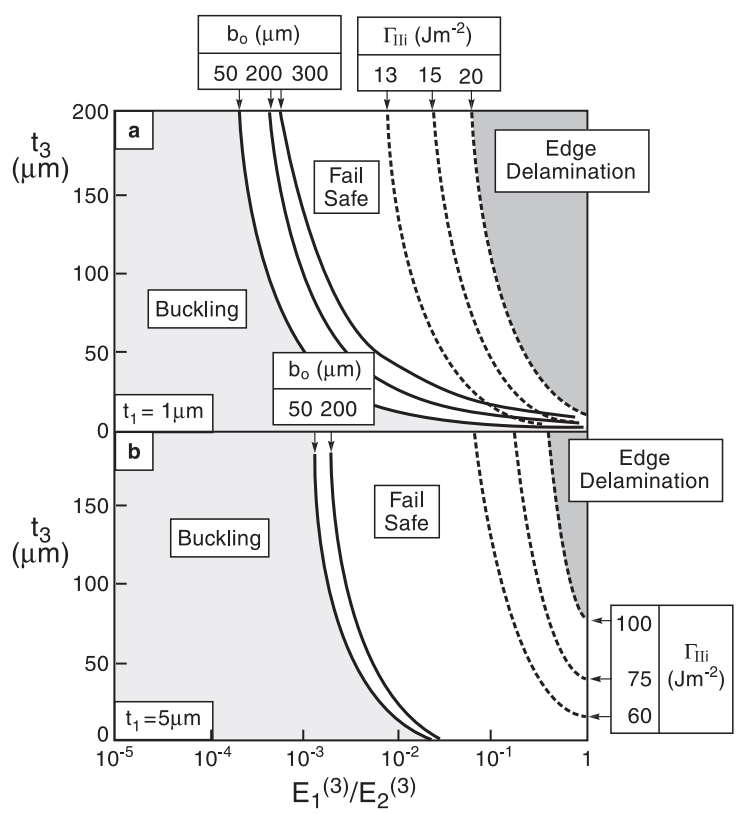

Fig. 12. Combinations of top coat thickness and modulus ratio such that neither buckling nor edge-delamination will occur for the TBC specified in Eq. (5.1). An acceptable combination, $\left(t_{3}, E_{1}^{(3)} / E_{2}^{(3)}\right)$, must lie between the solid line curve for the initial flaw size and the dashed line curve for the mode II interface toughness. (a) TGO $1 \mu \mathrm{m}$ thick, and (b) TGO $5 \mu \mathrm{m}$ thick. Note that even without a top coat, spontaneous delamination would occur if, in case (a) $\Gamma_{\mathrm{II} i}<12.5 \mathrm{~J} \mathrm{~m}^{-2}$, absent friction at the debonded interface, and, in case (b) if $\Gamma_{\mathrm{II} i}<56 \mathrm{~J} \mathrm{~m}^{-2}$. 
terface toughness. Note that for a $1 \mu \mathrm{m}$ TGO (Fig. 12(a)) $\Gamma_{\mathrm{II} i}$ must exceed $12.5 \mathrm{~J} \mathrm{~m}^{-2}$ to avert spontaneous edge delamination. Larger $\Gamma_{\mathrm{II} i}$ provide the possibility that the fail-safe domain identified on the figure can be realized. Fig. 12(b) presents a second illustration of the fail-safe combinations: in this case for a TBC system which is identical except that the thickness of the TGO layer (\#1) is now $5 \mu \mathrm{m}$. The greater thickness of the TGO layer requires higher interface toughness to resist spontaneous edge-delamination $\left(\Gamma_{\mathrm{II} i}>56 \mathrm{~J} \mathrm{~m}^{-2}\right)$, but the TBC is somewhat less susceptible to buckling in the presence of small initial delamination flaws. Note that for typical TBC moduli $\left(E_{1}^{(3)} / E_{2}^{(3)} \approx 10^{-1}\right)$, there is a wide fail safe range provided that $\Gamma_{\mathrm{II} i}$ exceeds $56 \mathrm{~J} \mathrm{~m}^{-2}$.

Erosion of the interface toughness or growth of initial delaminations by sub-critical mechanisms such as stress corrosion (Sergo and Clarke, 1998) or fatigue (Wang and Evans, 1998) will narrow, and perhaps even eliminate, the window of top coat properties capable of suppressing both buckling and edge-delamination.

\section{Summary}

The primary focus of the paper has been on the competition between edge- and buckle-delamination of the TBC at the $\mathrm{TGO} /$ bond coat interface, as influenced by the in-plane modulus of the top coat. Both forms of delamination scale with the available elastic energy per unit area stored in the tri-layer, $G_{0}$, given by Eq. (3.6). Suppression of edge-delamination requires that the effective mode II interface toughness exceed $G_{0}$. Buckling-delamination takes place as interface cracking under combined mode I and mode II and is therefore likely to experience lower interface toughness. The relatively thick top coat acts to completely suppress buckling whenever the initial interface flaws are smaller than a critical size. Detailed conditions on the in-plane modulus of the top coat for simultaneously suppressing both edge-delamination and buckling have been given.

\section{Acknowledgements}

This work was supported in part by the NSF through Grant NSF-CMS-96-34632, by the ONR through Grant N00014-96-10059 and by the Division of Applied Sciences, Harvard University.

\section{Appendix A}

\section{A.1. Bending and stretching stiffnesses of a multi- layered plate}

The location, $y=c$, of the neutral axis for pure bending about the $z$-axis of a plate of $J$ layers is given by (cf. Fig. 4)

$c \sum_{i=1}^{J} \bar{E}_{i} t_{i}=\frac{1}{2} \sum_{i=1}^{J} \bar{E}_{i}\left(y_{i+1}^{2}-y_{i}^{2}\right)$,

where $\bar{E}_{i}=E_{i} /\left(1-v_{i}^{2}\right)$ and $y_{i+1}$ and $y_{i}$ are the locations of the top and bottom faces of layer \# $i$. The bending and stretching stiffnesses introduced in Eq. (3.2) are given by

$$
\begin{aligned}
& S=\sum_{i=1}^{J} \bar{E}_{i} t_{i} \quad \text { and } \\
& B=\frac{1}{3} \sum_{i=1}^{J} \bar{E}_{i}\left[\left(y_{i+1}-c\right)^{3}-\left(y_{i}-c\right)^{3}\right] .
\end{aligned}
$$

\section{A.2. The Determination of $k$ for layer \#3 introduced} in Eq. (4.6)

In the notation of Eq. (4.3) for plane strain deformations in the $\left(x_{1}, x_{2}\right)$ plane, the Navier equations for the pre-stressed layer \#3 are

$$
\begin{aligned}
& \left(c_{11}-\sigma_{0}\right) u_{1,11}+c_{44} u_{1,22}+\left(c_{12}+c_{44}\right) u_{2,12}=0, \\
& \left(c_{12}+c_{44}\right) u_{1,12}+c_{22} u_{2,22}+\left(c_{44}-\sigma_{0}\right) u_{2,11}=0,
\end{aligned}
$$

where $\sigma_{0} \equiv \sigma_{0}^{(3)}$ is the compressive pre-stress in the $x_{1}$-direction and $u_{\alpha}\left(x_{1}, x_{2}\right)$ are the displacements measured from the pre-buckling state. The Eq. (A.3) have been obtained by linearizing the nonlinear equilibrium equations about the prebuckling state for material (4.3). The top of the 
layer at $x_{2}=t_{3}$ is traction-free requiring that the stress changes $\Delta \sigma_{22}=0$ and $\Delta \sigma_{12}=0$ vanish. On $x_{2}=0$,

$$
\begin{aligned}
& u_{1}=u_{2}=0 \quad \text { for }\left|x_{1}\right|>b, \\
& u_{1}=0, \quad u_{2}=(\delta / 2)\left[1+\cos \left(\pi x_{1} / b\right)\right] \\
& \text { for }\left|x_{1}\right|<b .
\end{aligned}
$$

The desired quantity $k$ defined in Eq. (4.6) is obtained in terms of the normal stress component on $x_{2}=0$ for $\left|x_{1}\right|<b$ by

$$
\begin{aligned}
k= & -\left(2 E_{1}^{(3)} \delta\right)^{-1} \int_{-b}^{b}\left(1+\cos \left(\pi x_{1} / b\right)\right) \\
& \times \Delta \sigma_{22}\left(x_{1}, 0\right) \mathrm{d} x_{1} .
\end{aligned}
$$

To lowest order, the condition $u_{1}=0$ along the interface where layer \#3 joins the bi-layer plate is consistent with the in-plane displacement of the plate vanishing in the buckling problem. This provides the elastic energy change in layer \#3 to order $\delta^{2}$, where $\delta$ is the buckling amplitude introduced in Eq. (4.5). This condition is not imposed on the plate itself.

The linear boundary value problem specified by Eqs. (A.3) and (A.4) is solved using a method which has been employed on similar problems by Sneddon (1951) and Delale and Erdogan (1979). A Fourier transform of the governing equations and boundary conditions it taken with respect to the $x_{1}$-coordinate leading to a set of ordinary differential equations and boundary conditions in the transformed variables in the $x_{2}$-coordinate. These can then be solved in closed form, and inverse transform expressions can be written for the solution quantities. In particular, the quantity needed to evaluate $k$ in Eq. (A.5), $\Delta \sigma_{22}\left(x_{1}, 0\right)$, can be obtained in the form

$$
\Delta \sigma_{22}\left(x_{1}, 0\right)=E_{1}^{(3)}(\delta / b) \int_{0}^{\infty} K(\eta) \cos \left(\eta x_{1} / b\right) \mathrm{d} \eta,
$$

where $K(\eta)$ is an explicit, but complicated, function of the moduli ratios, and of $\sigma_{0} / E_{1}^{(3)}$ and $t_{3} / b$. Numerical integration is used in the evaluation of both Eqs. (A.6) and (A.5).
The Poisson's ratio quantity $v_{12}^{(3)}$ in Eq. (4.2) can be taken to be zero, to very good approximation, in the case of interest where $E_{2}^{(3)} / E_{1}^{(3)} \gg$ 1 and the magnitude of the stress component $\sigma_{22}$ is not significantly larger than the magnitudes of the other components. The argument is as follows. Considering nonzero stress components $\sigma_{11}$ and $\sigma_{22}$, write $\varepsilon_{11}$ and $\varepsilon_{22}$ as

$\varepsilon_{11}=\frac{1}{E_{1}} \sigma_{11}-\frac{v_{21}}{E_{2}} \sigma_{22}, \quad$ and

$\varepsilon_{22}=-\frac{v_{12}}{E_{1}} \sigma_{11}+\frac{1}{E_{2}} \sigma_{22}$,

where by reciprocity $v_{21} / E_{2}=v_{12} / E_{1}$. Defined this way, both Poisson ratios will necessarily be less than 1 , which is all that is required for the present argument. If $E_{2} / E_{1} \gg 1$, the reciprocal relation then implies $v_{12} \cong 0$. Thus, in the computation of results for layer $\# 3$, we have taken $v_{12}^{(3)}=0$ in Eq. (4.2). The resulting coefficients in Eqs. (4.3) and (A.3) are then given by Eq. (4.4).

The asymptotic solution (4.7) for $k$ under conditions $E_{2}^{(3)} / E_{1}^{(3)} \gg 1$ and $\sigma_{0} / E_{1}^{(3)}=0$ is obtained by noting that, in the limit of large $E_{2}^{(3)} / E_{1}^{(3)}$, Eq. (A.3) becomes

$u_{1,11}+(\lambda / 2)\left(u_{1,22}+u_{2,12}\right)=0, \quad u_{2.22}=0$.

If $\Delta \sigma_{22}$ is comparable in magnitude to $\Delta \sigma_{11}$ and $\Delta \sigma_{12}$, then $\varepsilon_{22}$ will become zero as $E_{2}^{(3)} / E_{1}^{(3)}$ becomes large, implying with Eq. (A.8) that $u_{2}=f\left(x_{1}\right)$. Thus, by Eq. (A.4),

$$
\begin{aligned}
u_{2} & =f\left(x_{1}\right) \\
& = \begin{cases}(\delta / 2)\left(1+\cos \left(\pi x_{1} / b\right)\right), & \left|x_{1}\right|<b, \\
0, & \left|x_{1}\right|>b .\end{cases}
\end{aligned}
$$

Now, the first equation of (A.8) becomes

$u_{1,11}+(\lambda / 2) u_{1,22}=0$.

The remaining boundary conditions reduce to $u_{1}=0$ on $x_{2}=0, \quad$ and $u_{1,2}=-\mathrm{d} f\left(x_{1}\right) / \mathrm{d} x_{1}$ on $x_{2}=t_{3}$.

This asymptotic boundary value problem can be solved in closed form using the same transform method outlined for the full problem. The result for $k$ in Eq. (A.5) is given by Eq. (4.7) where 


$$
\begin{aligned}
H(z)= & \frac{\pi}{2} z-\frac{1}{2} \int_{0}^{\infty} \frac{\sinh (\eta z)}{\eta \cosh (\eta z)}\left(\frac{\sin (\pi+\eta)}{\pi+\eta}\right. \\
& \left.-\frac{\sin (\pi-\eta)}{\pi-\eta}\right)^{2} \mathrm{~d} \eta .
\end{aligned}
$$

The condition on the pre-stress for the applicability of the asymptotic formula (A.5) is $\sigma_{0} / E_{1}^{(3)} \ll\left(t_{3} / b\right)^{2}$.

\section{A.3. The coupled problem for the bi-layer plate and the compliant top layer}

Buckling of the straight-sided blister well behind the curved end is considered wherein the deflections and stresses are independent of the coordinate $x_{3}$ parallel to the edges. The notation introduced in Section 4 is used. Relations (3.2) hold for the bi-layer plate where $B$ and $S$ are evaluated from Eqs. (A.1) and (A.2) for $J=2$, and $N_{0}$ is given by Eq. (4.1). The plate is taken to be clamped along $x= \pm b$. Von Karman nonlinear strain-displacement relations are used to describe the plate. The additional strain of the neutral bending axis $\Delta \varepsilon$ and the curvature $\kappa$ are related to the in-plane and normal displacements $(u(x), w(x))$ of the neutral bending axis from the pre-stressed state by

$\kappa=w^{\prime \prime}, \quad \Delta \varepsilon=u^{\prime}+\frac{1}{2} w^{\prime 2}$,

where the prime denotes differentiation with respect to $x \equiv x_{1}$. Let $p(x)$ denote the normal restoring stress exerted by layer \#3 on the plate, and neglect the tangential restoring component, with justification to be given later. The equilibrium equations associated with Eq. (A.13) through the principle of virtual work are

$$
\Delta N^{\prime}=0, \quad \Delta M^{\prime \prime}+\left(N_{0}-\Delta N\right) w^{\prime \prime}=p .
$$

The first implies $\Delta N$ is independent of $x$. Then, Eq. (3.2), the second equation of (A.13) and the condition that $u$ vanishes at $x= \pm b$ gives

$$
\frac{\Delta N}{S}=\frac{1}{4 b} \int_{-b}^{b} w^{\prime 2} \mathrm{~d} x \text {. }
$$

The second equation of Eq. (A.14) with Eq. (3.2) and the first equation of (A.13) becomes

$$
B w^{\prime \prime \prime \prime}+\left(N_{0}-\Delta N\right) w^{\prime \prime}=p \text {. }
$$

Multiply Eq. (A.16) by $w$, and integrate both sides of the equation from $-b$ to $b$. Integration by parts, with the aid of the fact that $w$ and $w^{\prime}$ vanish at the ends of the interval, results in

$\int_{-b}^{b}\left[B w^{\prime \prime 2}-\left(N_{0}-\Delta N\right) w^{\prime 2}-p w\right] \mathrm{d} x=0$.

The solution to Eqs. (A.15) and (A.16) with $p=0$ is $w=(\delta / 2)[1+\cos (\pi x / b)]$ with $\delta$ related to $N_{0}$ through (A.15), as will be detailed below.

The plate is coupled to the compliant top layer by identifying $p$ with $\Delta \sigma_{22}\left(x_{1}, 0\right)$ in (A.6). Specifically, assuming an approximate solution in the form $w=(\delta / 2)[1+\cos (\pi x / b)]$, Eqs. (A.15) and (A.17) become

$$
\begin{aligned}
& \frac{\Delta N}{S}=\frac{1}{16}\left(\frac{\pi \delta}{b}\right)^{2}, \\
& N_{0}-\Delta N,=\left(\frac{\pi}{b}\right)^{2} B+\frac{4 k E_{1}^{(3)} b}{\pi^{2}},
\end{aligned}
$$

where the dependence of $k$ on the moduli ratios, $t_{3} / b$ and $\sigma_{0} / E_{1}^{(3)}$ have already been documented. This is the exact solution to the plate problem posed in the limit when the top layer is absent. The condition for the onset of buckling, Eq. (4.8), is obtained as the limit from Eq. (A.18) when $\delta$ is small. The moment change in the bi-layer plate at its right end can be computed from $\Delta M=B w^{\prime \prime}$ and is found to be

$\Delta M=\frac{\pi^{2}}{2} \frac{B \delta}{b^{2}}$.

The energy release rate for the interface crack tip of the buckled blister can be determined approximately in two ways, each of which is outlined below. In the first approach, it is noted that the right end of the bi-layer plate experiences the loading combination $(\Delta N, \Delta M)$. On the scale of the bi-layer thickness, one can regard this as a composite beam attached to a substrate (cf. Hutchinson and Suo, 1992). The crack tip stress intensities are due to $(\Delta N, \Delta M)$ because there is no 
intensity for the unbuckled plate under the prestress $N_{0}$. The energy release rate is

$G=\frac{\Delta M^{2}}{2 B}+\frac{\Delta N^{2}}{2 S}$.

Noting that $\Delta N=N_{0}-N_{\mathrm{c}}$, one can algebraically reduce (A.20) using Eqs. (A.18) and (A.19) to obtain the expression for $G$ given by Eq. (4.9).

One can also use this approach to obtain an approximation for the mode mixity. For an beam of one isotropic material attached to a substrate of another isotropic material, the mode mixity is given by

$\tan \psi=\frac{\sqrt{12}+(t \Delta N / \Delta M) \tan \omega}{-\sqrt{12} \tan \omega+(t \Delta N / \Delta M)}$,

where $t$ is the beam thickness and $\omega$ is a function of elastic mismatch. For elastic mismatches which are not very large $\omega$ differs from its value for the homogeneous case $\left(51.2^{\circ}\right)$ by no more than about $5^{\circ}$. Apply Eq. (A.21) to the bi-layer beam by taking the thickness to be that of an uniform beam with the same bending and stretching stiffness, i.e., $t=\sqrt{12 B / S}$, and, thus, $t \Delta N / \Delta M=$ $\sqrt{3\left(N_{0}-N_{\mathrm{c}}\right) /\left[B(\pi / b)^{2}\right]}$ from Eq. (A.19). The elastic mismatch has been ignored $\left(\omega=51.2^{\circ}\right)$ in plotting the mode mixity in Fig. 9.

The second approach to computing the energy release rate involves taking the derivative with respect to $b$ of the elastic energy change of the system relative to the pre-stressed state. It can be shown by direct calculation that the difference in the elastic energy in the plate and top layer between the buckled and unbuckled states is exactly $\Delta U=-b \Delta N^{2} / S$ for the model posed. This accounts for the elastic energy change in the top layer. A direct calculation of the energy release rate from $G=-1 / 2 \mathrm{~d} \Delta U / \mathrm{d} b$ gives

$$
\begin{aligned}
G= & \frac{1}{2 S}\left[N_{0}-N_{\mathrm{c}}\right] \\
& \times\left[N_{0}+3 N_{\mathrm{c}}-\frac{8 k}{\pi^{2}} E_{1}^{(3)} b\left(3-\frac{z}{H} \frac{\mathrm{d} H}{\mathrm{~d} z}\right)\right],
\end{aligned}
$$

where asymptotic expression (4.7) for $k$ has been used and $z=\sqrt{2 / \lambda}\left(t_{3} / b\right)$. This result differs slightly from that in Eq. (4.9). However, because $(z / H) \mathrm{d} H / \mathrm{d} z$ approaches 1 for values of $z$ larger than about 1 (cf. Fig. 6(b)), Eq. (A.22) reduces to Eq. (4.9) for $z>1$ and the difference at smaller $z$ is small. The examples in the body of the paper were computed using Eq. (4.9).

\section{References}

Christensen, R.J., Lipkin, D.M., Clarke, D.R., 1996. The stress and spalling behavior of the oxide scale formed on polycrystalline $\mathrm{Ni}_{3} \mathrm{Al}$. Acta Mater. 44, 2813-3821.

Christensen, R.J., Tolpygo, V.K., Clarke, D.R., 1997. The influence of the reactive element ytrium on the stress in alumina scales formed by oxidation. Acta Mater. 45, 1761-1766.

DeMasi-Marcin, J.T., Sheffler, K.D., Bose, S., 1989. Mechanisms of degradation and failure in a plasma deposited thermal barrier coating. ASME Paper 89-GT-132, American Society of Mechanical Engineering, New York.

Delale, F., Erdogan, F., 1979. Bonded orthotropic strips with cracks. Int. J. Fracture 15, 343-364.

Gioia, G., Ortiz, M., 1997. Delamination of compressed thin films. Adv. Appl. Mech. 33, 120-192.

He, M.Y., Evans, A.G., Hutchinson, J.W., 1998. Effects of morphology on the decohesion of compressed thin films. Mater. Sci. Engrg. A 245, 168-181.

Hillery, R., 1996. Coatings for High Temperature Structural Materials. National Research Council, National Academy Press, Washington, DC.

Hutchinson, J.W., Jensen, H.M., 1990. Models of fiber debonding and pullout in brittle composites with friction. Mech. of Mater. 9, 139-163.

Hutchinson, J.W., Suo, Z., 1992. Mixed mode cracking in layered materials. Adv. Appl. Mech. 29, 63-191.

Johnson, C., Ruud, J.A., Kaya, A.C., Lorenzi, H.G., 1995. In: Proceedings of the Eighth National Thermal Spray Conference. pp. 415-420.

Lee, E.Y., Sisson, R.D., 1994. The effect of bond coat oxidation on the failure of thermal barrier. In: Berndt, C.C., Sampath, S. (Eds.), Proceedings of the Seventh National Thermal Spray Conference. Materials Park, ASM International, Ohio, pp. 55-59.

Nilsson, K.F., Thesken, J.C., Sindelar, P., Giannakopoulos, A.E., Storåkers, B., 1993. A theoretical and experimental investigation of buckling induced delamination growth. J. Mech. Phys. Solids 41, 749-782.

Sergo, V., Clarke, D.R., 1998. Observation of sub-critical spall propagation of a thermal barrier coating. J. Amer. Ceram. Soc. 81, 3237-3242.

Sneddon, I.N., 1951. Fourier Transforms. McGraw-Hill, New York.

Thouless, M.D., Hutchinson, J.W., Liniger, E.G., 1992. Planestrain, buckling-driven delamination of thin films: Model experiments and mode II fracture. Acta Metall. Mater. 40, 2639-2649. 
Thouless, M.D., Jensen, H.M., Liniger, E.G., 1994. Delamination from edge flaws. Proc. R. Soc. Lond. A 447, 271-279.

Wang, J.S., Evans, A.G., 1999. Effects of strain cycling on buckling, cracking and spalling of a thermally grown alumina on a nickel-based bond coat. Acta Mater. 47, 699710 .
Wang, J.S., Evans, A.G., 1998. Measurement and analysis of buckling and buckle propagation in compressed oxide layers on superalloy substrates. Acta Mater. 46, 49935005 . 